\title{
RESEARCH ON REMOTE CROP PRODUCTION MANAGEMENT SYSTEM BASED ON CROP SIMULATION MODELS AND WEBGIS
}

\author{
Jianbing Zhang ${ }^{1, *}$, Yeping Zhu ${ }^{2}$, Liping Zhu ${ }^{1}$ \\ ${ }^{1}$ Department of Computer Science and Technology, China University of Petroleum-Beijing, \\ Beijing, China, 102249 \\ ${ }^{2}$ Institute of Agricultural Information, Chinese Academy of Agricultural Sciences, Beijing, \\ China, 100081 \\ * Corresponding author, Address: Department of Computer Science and Technology, China \\ University of Petroleum-Beijing, 18 Fuxue Road, Changping, Beijing, P. R. China, 102249, \\ Tel: +86-10-89733006, Email: zhangbing153@yahoo.com.cn
}

Abstract: In order to simulate growth and yield of crop in field scale, crop simulation models always require a lot of initial parameters. WebGIS is a powerful tool for obtaining, managing and analyzing spatial data on the Web. With the support of WebGIS, crop models based on web services can be easily run on the web by remote client and provide decision information and measures for crop production. In this paper, a remote crop production management system based on crop models and WebGIS is discussed. After the input variables for the crop model are obtained by WebGIS tool, the model will be run and the database will be updated with the results of the simulation. Spatial analysis and representation of crop model input data and output results can be done effectively on the web. Both the input data for the model and outputs from the model can be displayed on a map.

Keywords: crop model, WebGIS, crop production

\section{INTRODUCTION}

Crop production, farm management and decision making are influenced by a lot of uncertain factors, such as biological and technological ones. Some important decisions in agricultural production, such as irrigation application 
dates and amount, fertilizer application dates and amount, the choose of crop variety are depended heavily on the existing knowledge base of current environment conditions like soil and climate, water resource (Wang Shiqi, 1998). Cropping inputs such as fertilizer are applied at varying rate across a field in response to variations in crop needs. Spatial variability of crop production such as different soil conditions, weather conditions and water conditions needs different agricultural production management practices within a target-region.

Crop model simulates growth and yield of crop in field scale and can be used to provide useful field information for farmers. Due to the complexity of crop-environment system, the limit of agricultural data collection and some technology restrictions, crop model techniques have not been widely applied in agricultural production. Researchers have used crop models for many years, but have had limited success in packaging these complex models in a framework that make them easy for producers to use.

Web-based applications provide an efficient and powerful way for delivering crop models to crop producer. An important issue in crop simulation model is that the basic units (water, soil and weather data) have a spatial distribution. WebGIS is a powerful tool for spatial data management on the web. With the support of WebGIS tool, crop models can be easy for agricultural producers as well as policy makers to use via the Internet.

This paper describes the application of integration WebGIS with webbased crop models for crop production management. An integrated system of WebGIS and crop modeling helps people know the impact of differences between input and output spatially from one place to other, provide better management information from productivity and profitability viewpoint quickly. The design and implementation of the remote crop production management system are also introduced in this paper.

\section{CROP SIMULATION MODEL}

Crop model simulates growth and yield of crop in field scale, and requires a lot of initial conditions and parameters as input data such as soil, water, climate data etc. There are many crop models nowadays in the world. But few can be run on the web. In order to implement remote crop production management, we need the model to be run on the web.

Intelligent Wheat and Corn Management System is the result of many years' research in wheat and corn simulation by Yeping zhu, Shiqi Wang in CAAS (Chinese Academy of Agricultural Science). The research was supported by Chinese Academy of Agricultural Science and National 863306 project of China.

The wheat and corn models simulate growth and yield of crop in field scale, and they also require initial conditions and parameters. The inputs for 
the model include environmental conditions (soil type, daily maximum and minimum temperature, rainfall, and solar radiation) and management practices (variety, row spacing, plant population). The simulation result includes daily growth of vegetative, stage, and water and nitrogen stress. Some decision information can also be given to assist producers in their management.

In order to be used on the web environment, wheat and corn crop models are both packaged with web service and XML technology. Web service is a technology that designed to support interoperable Machine to Machine interaction over a network. Web services are frequently just Web APIs that can be accessed over a network, such as the Internet, and executed on a remote system hosting the requested services. By using web services technology, the wheat and corn models can be run on the web by remote client. The data exchange format is XML.

\section{WEBGIS}

WebGIS is a powerful tool for obtaining, managing and analysing spatial data on the Internet. WebGIS holds the potential to make distributed geographic information available to a very large worldwide audience.

It is possible to add GIS functionality to web-based crop model applications by WebGIS tool. It makes data access including model inputs and model results more conveniently. Both the input data for the model and outputs from the model can be displayed on a map. It helps effective use of GIS for the spatial analysis and representation of input data and output results of crop model. By this way, we can get a more clear impression on the spatial distribution of those environment elements and simulation results.

GeoBeans software is WebGIS software which is developed by network technology department, Institute of Remote Sensing Application, CAS. In our research, we use GeoBeans Map Server for map function and model data management.

\section{DESIGN AND IMPLEMENTATION OF REMOTE CROP PRODUCTION MANAGEMENT}

\subsection{Design of the system}

In order to make the system more robust, flexible, we adopt the popular three-tier architecture in our system design. The architecture of the system is 
composed of three layers: the user interface layer, the application logic layer and the database layer. The architecture makes our development and integration work easily and efficiently.

Before users run the model on the Internet, the crop variety and simulation location must be pointed out. For each cell of the grid where remote client lives on, WebGIS tool will extract the input variables (climate, crop, soil, ...) for the crop model from the corresponding data layers, then the model will be run and the database will be updated with the results of the simulation.

The workflow and architecture of the system are as follow:

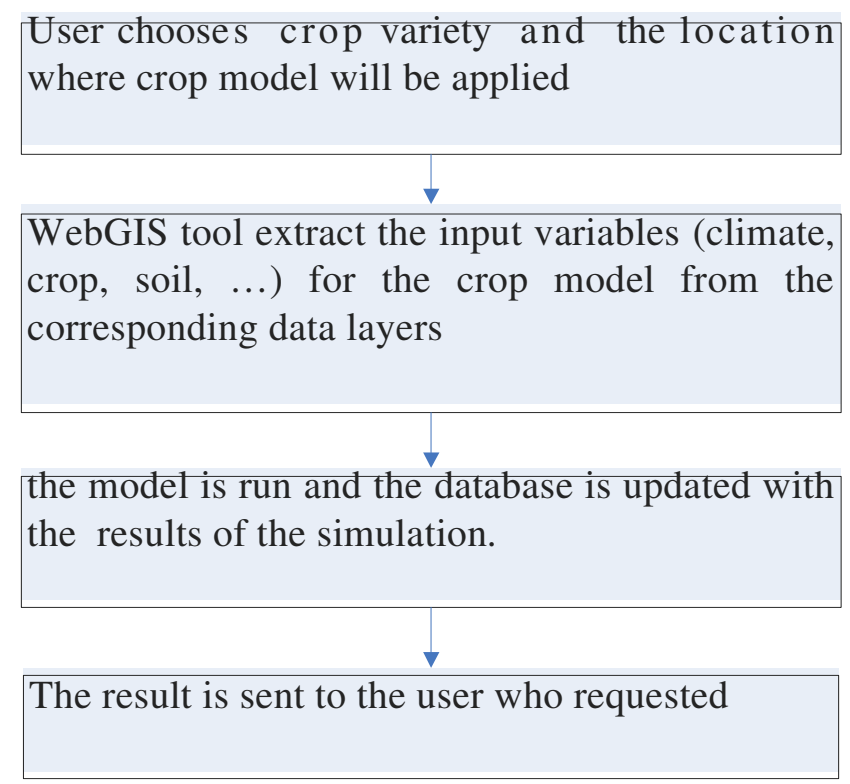

Fig. 1. showing the work flow of the system

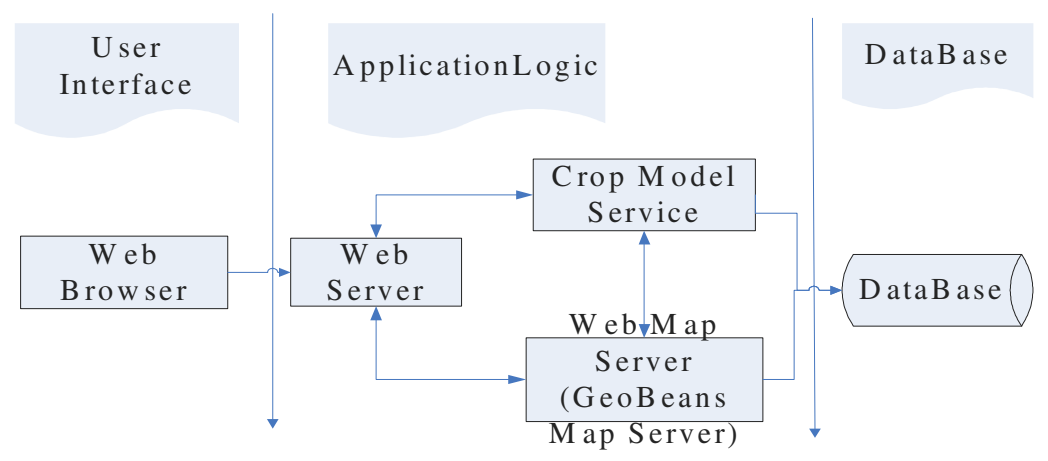

Fig. 2. Showing the architecture of the system 


\subsection{Implementation}

The remote crop management system is implemented by the integration of WebGIS and crop simulation model. The communication between WebGIS service and crop model services is the key part of the system. Web services, XML technology are applied to the communication. XML is exchange data format.

After connecting WebGIS and crop models successfully, users can run the model easily through internet in their convenience. The delivering crop models to crop producer has achieved in our system. It helps crop producer a lot to know the impact of different measurement in crop production.

Some of running results of the system are as follow:

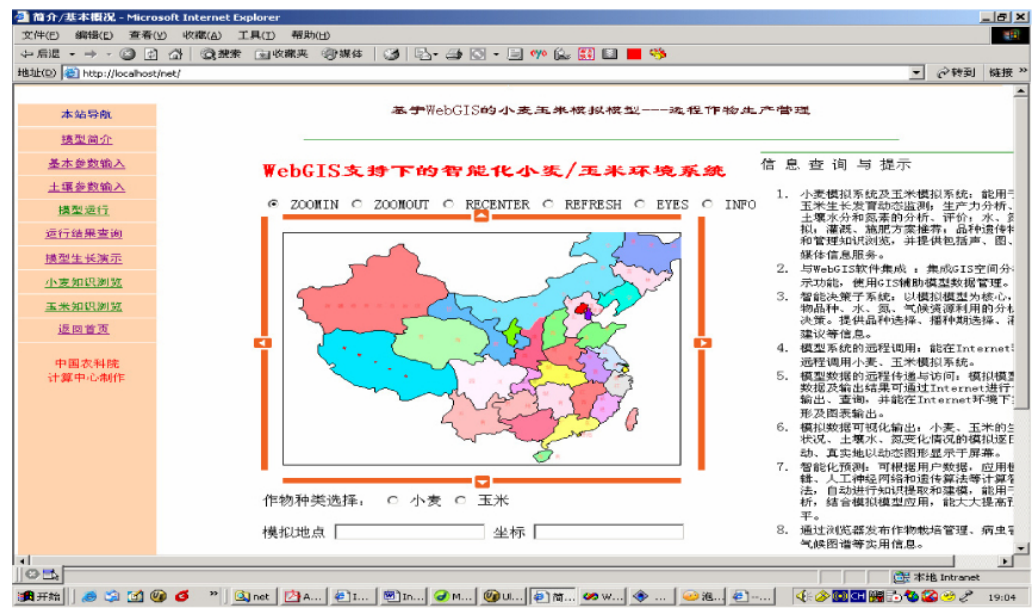

Fig. 3. Showing the main window of the system

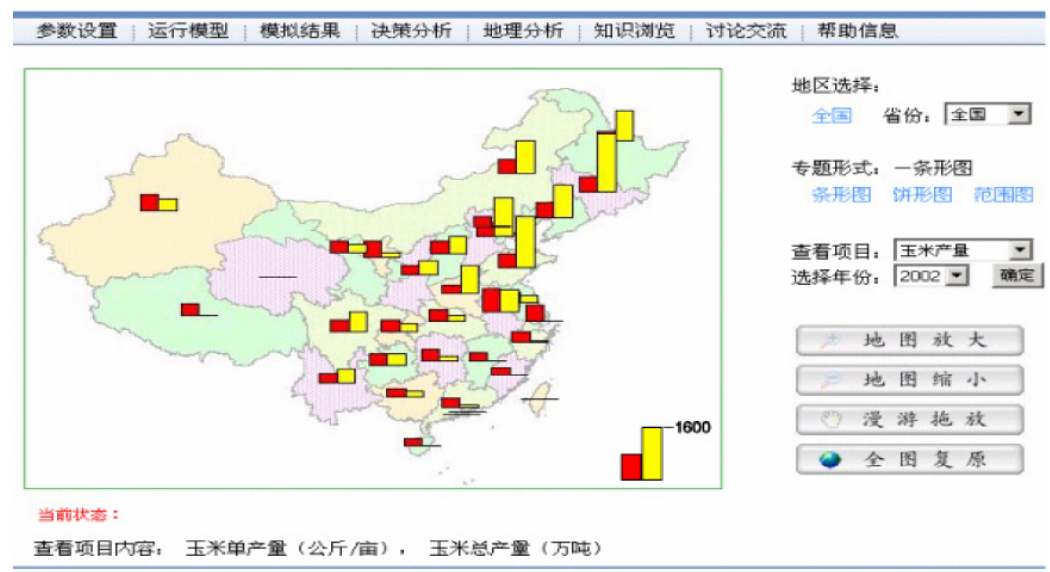

Fig. 4. Showing simulation results on the map 


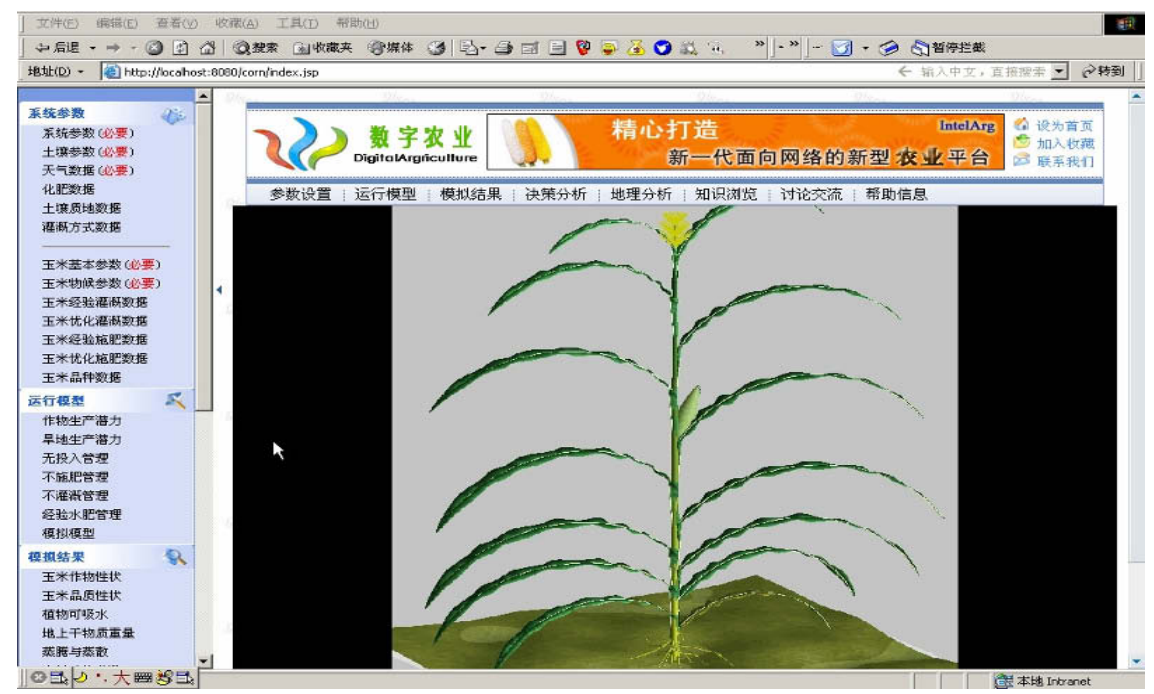

Fig. 5. Showing corn simulation results

\section{CONCLUSIONS}

WebGIS based crop models are expected to give a new approach in order to provide agricultural managers with a powerful tool to assess simultaneously the effect of farm practices to crop production. In this study, a system framework is designed using three-tier architecture, while crop models are packaged with web services and Geobeans software acts as a pre and post processor for model data. The graphical display of inputs and outputs of wheat and corn model (CAAS) are also supported by Geobeans Map Server. Spatial analysis and representation of crop model input data and output results can also be done effectively by the system.

The study successfully demonstrated the integration of crop model with WebGIS and its application in remote crop production management. With the support of WebGIS tool, crop models will be easy to use in crop production.

\section{ACKNOWLEDGEMENTS}

This research was supported by National Scientific and Technical Supporting Programs Funded by Ministry of Science and Technology of China (2006BAD10A06), and Digital Agriculture Program of State Hightech Research and Development Project of China (No. 20060110Z2059). 


\section{REFERENCES}

Engel T. AEGIS/WIN: A computer program for the application of crop simulation models across geographic areas [J]. Agron. J. 1997, 89(6):919-928.

Pan Xuebiao. Study on the Spatial Distribution and Variation of Cotton Production in Counties of China Based on GIS. Scientica Agricultura Sinica, 2003, 36(4):382-386.

Pan Yuchun, Wang Jihua. WebGIS-based system for crop quality monitoring and planting optimization. Transactions of the Chinese Society of Agricultural Engineering, 2004, 20(6):120-123.

Paz, JO, WD Batchelor. Web-based soybean yield simulation model to analyze the effects of interacting yield-limiting factors variations of winter time air pollution concentrations in the city of Graz, Austria. Environmental Monitoring and Assessment, 2001, 65:79-87.

Wang hong, Wu Shuan, Tang zhenghong. Crop productivity model and its application. Chinese Journal of Applied Ecology, 2002, 13(9):1174-1178.

Wang Shiqi Zhu Yeping etc. A System Framework Based on Crop Simulator. Modeling for Crop-Climate-Soil-Pest System and Its Applications in Sustainable Crop Production 1998, 6 . 